\title{
Article \\ Public KAP towards COVID-19 and Antibiotics Resistance: A Malaysian Survey of Knowledge and Awareness
}

\author{
Chee Tao Chang ${ }^{1 \oplus}$, Ming Lee ${ }^{2}$, Jason Choong Yin Lee ${ }^{3}{ }^{\oplus}$, Nicholas Chor Teng Lee ${ }^{4}$, Tsu Yin $\mathrm{Ng}^{5}$, \\ Asrul Akmal Shafie ${ }^{6, *(D)}$ and Kah Shuen Thong ${ }^{4}$ \\ 1 Clinical Research Centre, Hospital Raja Permaisuri Bainun, Ministry of Health Malaysia, \\ Ipoh 30450, Malaysia; davidcct.crc@gmail.com \\ 2 Klinik Kesihatan Kampung Simee, Ministry of Health Malaysia, Ipoh 31400, Malaysia; \\ 105leeming@gmail.com \\ 3 Perak Pharmaceutical Services Division, Ministry of Health Malaysia, Tanjung Rambutan 31250, Malaysia; \\ jasonlcy@gmail.com \\ 4 Pharmacy Department, Hospital Raja Permaisuri Bainun, Ministry of Health Malaysia, Ipoh 30450, Malaysia; \\ nlct86@gmail.com (N.C.T.L.); kahshuenthong@yahoo.com (K.S.T.) \\ 5 Klinik Kesihatan Taiping, Perak, Ministry of Health Malaysia, Taiping 30400, Malaysia; \\ tsuyinng@hotmail.com \\ 6 School of Pharmaceutical Sciences, University Sains Malaysia, Gelugor 11800, Malaysia \\ * Correspondence: aakmal@usm.my
}

check for updates

Citation: Chang, C.T.; Lee, M.; Lee, J.C.Y.; Lee, N.C.T.; Ng, T.Y.; Shafie, A.A.; Thong, K.S. Public KAP towards COVID-19 and Antibiotics Resistance: A Malaysian Survey of Knowledge and Awareness. Int. J. Environ. Res. Public Health 2021, 18, 3964. https://doi.org/10.3390/ ijerph18083964

Academic Editor: Paul B. Tchounwou

Received: 16 March 2021

Accepted: 7 April 2021

Published: 9 April 2021

Publisher's Note: MDPI stays neutral with regard to jurisdictional claims in published maps and institutional affiliations.

Copyright: (c) 2021 by the authors. Licensee MDPI, Basel, Switzerland. This article is an open access article distributed under the terms and conditions of the Creative Commons Attribution (CC BY) license (https:// creativecommons.org/licenses/by/ $4.0 /)$.

\begin{abstract}
This study aimed to assess the knowledge of the Malaysian public on the coronavirus disease 2019 (COVID-19) and antibiotics, the practice of preventive measures and attitude towards the new norms. The web-based questionnaire was disseminated online from 1 to 31 October 2020. Out of 2117 respondents, 1405 (66.4\%) knew that transmission of COVID-19 virus could happen in asymptomatic people. In term of antibiotics knowledge, $779(36.8 \%)$ respondents were aware that taking antibiotics could not speed up the recovery process of all infections. Less than half of the respondents $(49.0 \%)$ knew that antibiotics are effective against bacterial infection only. Majority (92.3\%) practiced good preventive measures. Majority of the respondents strongly agreed that quarantine should be made mandatory for all arrival from overseas $(97.2 \%)$ and wearing face masks should be made mandatory in all public areas $(94.0 \%)$. Respondents of Chinese ethnicity $(p=0.008)$, middle-aged $(p=0.002)$, with tertiary education $(p=0.015)$ and healthcare related education $(p<0.001)$, from the higher income groups $(p=0.001)$ were more likely to have better knowledge on COVID-19. The Malaysian public demonstrated good knowledge towards COVID-19, adequate practice of preventive measures and high acceptance towards the new norm. Knowledge on antibiotics use and resistance was poor, which warrants attention from the health authorities.
\end{abstract}

Keywords: COVID-19; antibiotic resistance; KAP survey model; preventive measures; Malaysia

\section{Introduction}

The coronavirus disease 2019 (COVID-19) was declared as a pandemic by the World Health Organization (WHO) in March 2020. Until 15 November 2020, there were cumulatively 53.7 million confirmed cases and 1.3 million deaths reported to the WHO [1]. The COVID-19 virus is known to be transmitted through respiratory droplets produced when an infected person coughs, sneezes, talks, or breathes [2]. While COVID-19 has influenzalike symptoms, previous reports suggested it had caused higher rate of transmission, hospitalization and fatality than influenza [3].

In Malaysia, the first case of COVID-19 was reported on 25 January 2020 [4]. The number of positive cases passed the 500 mark on 16 March 2020 [4]. To contain the virus transmission, the Malaysian government implemented the Movement Control Order (MCO) on 18 March 2020 [5]. To date, Conditional Movement Control Order (CMCO) was reinforced in targeted areas with high number of COVID-19 cases [6]. The public had 
to adapt to the new normal including mandatory wearing of face masks at public areas, practice physical distancing, frequent hand-washing and restrictions on mass gatherings [7]. These preventive directives were widely promoted as they were reported to be effective in curbing the infection $[8,9]$.

The knowledge, attitudes and practices (KAP) of the public are very important in this context and were widely studied after the COVID-19 outbreak [10]. In China, the general public demonstrated good knowledge, positive attitudes and appropriate practices in taking preventive measures [11]. Similar findings were found in Palestine, Saudi Arabia and Vietnam studies [12-14]. In contrast, lower income countries such as Syria, Pakistan, Bangladesh and Philippines reported lower KAP scores [15-18]. A local study conducted in Malaysia during the early stages of MCO found that the general public had good knowledge and positive attitudes towards the control of COVID-19, except for the practice of wearing face masks [19]. Another study in Australia reported adequate public knowledge on hand hygiene and physical distancing, but awareness on the use face mask and antibiotics remained insufficient [20]. A systematic review reported that the overall public KAP on COVID-19 was good in most countries, except a few in the initial stage of the pandemic [10].

The large increase in COVID-19 cases may lead to misuse of antibiotics. A metaanalysis of 24 studies reported that while the proportion of COVID-19 patients co-infected by bacterial source was low $(6.9 \%)$, a majority of them $(71.9 \%)$ received antibiotics [21]. In Malaysia, previous local studies found that the general public commonly believed antibiotics could be used to treat viral infections and expected physicians to prescribe antibiotics when they suffered from flu-like symptoms [22,23]. Such strongly held beliefs may further contribute to the misuse of antibiotics during this pandemic [24].

KAP surveys can identify knowledge gaps, cultural beliefs, or behavioural patterns that may facilitate understanding and action, as well as pose problems or create barriers development efforts. This can also serve as an important source of information for policymakers to design educational materials tailored to specific knowledge gaps among the public. While KAP studies had been conducted throughout the COVID-19 pandemic, knowledge of the general public on antibiotics use and resistance was not reported by previous COVID-19 KAP studies [10]. Moreover, most of these studies were conducted at the early stage of pandemic outbreak $[11,19]$. Hence, a follow-up study would be timely to measure the sustainability and changes in KAP over time. This study aims to determine the knowledge, attitude and practice related to COVID-19 and antibiotics use amongst the general public in Malaysia.

\section{Materials and Methods}

This was a cross-sectional study conducting using an online questionnaire via Google Forms. Malaysian residents were invited to take part in this survey. The link (URL) of the online questionnaire was distributed via email, mobile chat applications (e.g., WhatsApp, Telegram) and social media (e.g., Facebook, Instagram) to the target participants in October 2020 for one month. Residents who were above 18 years of age and stayed within the country since March 2020 were eligible to participate. Questions confirming the age and residential area were asked before the respondents were directed to the questionnaire page. Malaysian citizens staying permanently in other countries were excluded.

The respondents were sampled using convenience and snowball techniques. The researchers created a one-page recruitment banner and used the Ministry of Health Malaysia Facebook page as the main platform for questionnaire distribution. Personal and professional networks of the researchers were also used to maximize participation. Sample size was estimated using Raosoft online sample size calculator based on the formula for estimation of a proportion with finite population correction and using a conservative value of $50 \%$ of the population willing to wear a face mask, based on the findings by Azlan et al. [17] as the desired proportion to be estimated. This was the most conservative approach to generate the minimum sample size required for a survey-based study. With a precision of 0.025 , a total of 1537 samples were required for this study. 
The investigators developed the questionnaire in the national language (Malay) with reference to relevant literature [11-20,22,23]. It consisted of five sections: (i) demographic characteristics, (ii) knowledge of COVID-19 pandemic (7 items), (iii) preventive measures during COVID-19 pandemic (11 items), (iv) knowledge of antibiotics use and resistance (10 items) and v) attitude towards new norms during the COVID-19 pandemic (7 items). The responses in both the knowledge domains were categorised into "true", "false" and "unsure". Responses in the practice domain were categorized into "yes" or "no". Responses in the attitude domain were measured based on a 5-point Likert scale from strongly disagree, disagree, neutral, agree to strongly agree (Appendix A).

The first draft of the questionnaire underwent face validation and content validation by one public health specialist, one infectious disease specialist and one senior clinical pharmacist to examine its (i) relevance, (ii) clarity and (iii) comprehensiveness. The content reviewers examined every question in the questionnaire draft and commented whether the question was (i) relevant and (ii) easy to understand. If the particular question was not relevant, the content reviewer will either suggest amending or removing the question which they felt problematic. If the question was unclear, the reviewer will make suggestions on paraphrasing to improve clarity. After reviewing each domain, the content reviewer then assessed the (iii) comprehensiveness, whether the important aspects within that particular domain were covered. Additional questions were suggested to improve the comprehensiveness of the domain whenever appropriate. Once satisfied, reviewers proceeded to review the subsequent domains by repeating the aforesaid steps. The suggestions from the content reviewers were then reconciled by the investigators and the questionnaire was revised accordingly before the pre-testing stage.

The questionnaire then underwent pre-testing on 10 respondents from the general public to check clarity and understanding. Comments from the respondents were reconciled and further modifications were made. A pilot test was subsequently conducted on 30 respondents to examine the reliability and validity of the questionnaire. The tool demonstrated good internal consistency, with Cronbach's alpha values ranging from $0.712-0.861$ for the domains. The questionnaire was finalised after the pilot test.

This study was registered in the National Medical Research Registry (NMRR) and ethics approval was obtained from the Medical Research Ethical Committee (MREC) before the study commenced. Participants accessed the online questionnaire through a unique URL. Prior to answering the questionnaire, participants were directed to the participant information sheet, which consisted of details regarding the study. Respondents were informed that their participations were voluntary and their responses would be confidential. Participants who did not agree to participate were directed to the exit page. Those who consented voluntarily would click "agree to participate" and directed to the online questionnaire. Each participant was expected to complete the questionnaire within 15 to $20 \mathrm{~min}$. All responses were saved to a secure online database.

\section{Statistical Analysis}

The data analysis was performed using the Statistical Package for the Social Sciences (SPSS) for Windows (version 20.0; IBM, New York, NY, USA) and with statistically significant level set at 5\%. All the variables were analysed descriptively. The continuous variables were presented as means and standard deviation while the categorical variables were presented as frequency and percentages.

The responses in both the knowledge domains were re-categorised into "correct", "incorrect" and "unsure". Each correct response was given 1 point, and no point was given to incorrect/unsure responses. Each "yes" response in the practice domain was given 1 point. For the attitude domain, each agree or strongly agree responses were given 1 point. The minimum and maximum score range for each domain was as follows: knowledge on COVID-19 (0-7), knowledge on antibiotics (0-10), practice (0-11) and attitude (0-7). An arbitrary score of more than $80 \%$ in each domain would indicate good knowledge, appropriate practice and positive attitude. 
Independent samples t-test and one-way analysis of variance (ANOVA) were used to ascribe the KAP mean scores and subsequently differences across respondents with different sociodemographic characteristics (Appendix B). Univariate logistic regression was performed initially, and variables with $p<0.25$ were included into the multivariate logistic regression model. Multivariate logistic regression was then performed to determine the predictors of good knowledge towards COVID-19 and antibiotics, attitudes and practice. This was presented with adjusted odds ratios, $95 \%$ confidence interval and $p$-value. Pearson's correlation was performed to determine the relationship between knowledge scores on COVID-19, knowledge scores on antibiotics, practice scores and attitude scores.

\section{Results}

Out of 2217 responses, 55 did not fulfill the inclusion criteria and 45 refused consent. 2117 responses were analysed. The average age of the respondents was 32.96 years $(\mathrm{SD}=7.69$, range $=18-68)$, mostly resided in the Central Malaysia $(844,40.1 \%)$. Majority of the respondents were female $(1546,73.0 \%)$, Malay $(1381,65.2 \%)$, had tertiary education $(1870,88.3 \%)$, with no medical education background $(1527,72.1 \%)$, and with no chronic medical illness $(1844,87.1 \%)$. Characteristics of respondents were described in Table 1.

Table 1. Demographic characteristics $(n=2117)$.

\begin{tabular}{|c|c|c|}
\hline Characteristics & Frequency & Percentage \\
\hline \multicolumn{3}{|l|}{ Age (years) } \\
\hline $18-29$ & 736 & 34.8 \\
\hline $30-49$ & 1307 & 61.7 \\
\hline Above 50 & 74 & 3.5 \\
\hline \multicolumn{3}{|l|}{ Gender } \\
\hline Male & 571 & 27 \\
\hline Female & 1546 & 73 \\
\hline \multicolumn{3}{|l|}{ Ethnicity } \\
\hline Malay & 1381 & 65.3 \\
\hline Chinese & 430 & 20.3 \\
\hline Indian & 117 & 5.5 \\
\hline Others & 189 & 8.9 \\
\hline \multicolumn{3}{|l|}{ Education } \\
\hline Primary or below & 7 & 0.3 \\
\hline Secondary & 240 & 11.3 \\
\hline Tertiary & 1870 & 88.4 \\
\hline \multicolumn{3}{|l|}{ Occupation } \\
\hline Full time (government) & 730 & 34.5 \\
\hline Full time (private) & 915 & 43.2 \\
\hline Student & 151 & 7.1 \\
\hline Unemployed & 303 & 14.3 \\
\hline Retiree & 18 & 0.9 \\
\hline \multicolumn{3}{|c|}{ Medical education background } \\
\hline Yes & 590 & 27.9 \\
\hline No & 1527 & 72.1 \\
\hline \multicolumn{3}{|l|}{ Chronic medical illness } \\
\hline Yes & 273 & 12.9 \\
\hline No & 1844 & 87.1 \\
\hline \multicolumn{3}{|l|}{ Household income } \\
\hline Below RM 4850 & 1038 & 49 \\
\hline RM 4850 to RM 10,970 & 793 & 37.5 \\
\hline RM 10,971 and above & 286 & 13.5 \\
\hline
\end{tabular}


Table 1. Cont.

\begin{tabular}{ccc}
\hline Characteristics & Frequency & Percentage \\
\hline Region $^{\text {a }}$ & & \\
Central $^{* *}$ & 844 & 40.1 \\
Northern $^{*}$ & 563 & 26.7 \\
Southern $^{* * * *}$ & 250 & 11.8 \\
Eastern $^{* * *}$ & 184 & 8.7 \\
Sabah/Sarawak/Labuan & 269 & 12.7 \\
\hline
\end{tabular}

Note: Northern region consist of Perlis, Kedah. Pulau Pinang and Perak, ${ }^{* *}$ Central region consist of Selangor Wilayah Persekutuan and Negeri Sembilan, ${ }^{* * *}$ Eastern region consist of Pahang, Terengganu and Kelantan, **** Southern region consist of Melaka and Johor (Tamrin SB, Yokoyama K, Jalaludin J, Aziz NA, Jemoin N, Nordin R, Li Naing A, Abdullah Y, Abdullah M. The Association between risk factors and low back pain among commercial vehicle drivers in peninsular Malaysia: a preliminary result. Ind Health. 2007 Apr; 45(2):268-78, doi:10.2486/indhealth.45.268. PMID: 17485871). ${ }^{\text {a }} 7$ missing data, $n=2110$.

\subsection{Knowledge on COVID-19}

Seven questions were used to assess the knowledge of the respondents on COVID-19. The mean knowledge score was $6.36(\mathrm{SD}=0.87$, range $0-7)$. The overall proportion of correct answers was $90.9 \%(6.36 / 7 \times 100)$. Majority of the respondents were able to answer 6 out of 7 questions correctly $(1829,86.4 \%)$. Nevertheless, only two-thirds $(1405,66.4 \%)$ of the respondents knew that transmission of COVID-19 can happen even when a person did not develop symptoms. $343(16.2 \%)$ were unsure whether COVID-19 virus strain can mutate over time (Table 2). Differences in COVID-19 knowledge scores were observed across genders, age groups, ethnicity, educational level, occupation, medical education, household income and region (Table 3).

Table 2. Knowledge on COVID-19 $(n=2117)$.

\begin{tabular}{|c|c|c|c|c|}
\hline No. & Statement & Correct & Incorrect & Unsure \\
\hline 1. & The COVID-19 pandemic is of virus origin & $\begin{array}{l}2085 \\
(98.5)\end{array}$ & $\begin{array}{c}10 \\
(0.5)\end{array}$ & $\begin{array}{c}22 \\
(1.0)\end{array}$ \\
\hline 2. & $\begin{array}{l}\text { The main clinical symptoms of COVID-19 are fever, } \\
\text { cough, sore throat and difficulty in breathing }\end{array}$ & $\begin{array}{c}2102 \\
(99.3)\end{array}$ & $\begin{array}{c}5 \\
(0.2)\end{array}$ & $\begin{array}{c}10 \\
(0.5)\end{array}$ \\
\hline 3. & COVID-19 is highly contagious & $\begin{array}{l}2100 \\
(99.2)\end{array}$ & $\begin{array}{c}12 \\
(0.6)\end{array}$ & $\begin{array}{c}5 \\
(0.2)\end{array}$ \\
\hline 4. & $\begin{array}{l}\text { Elderly, children, people with co-morbidities and } \\
\text { immunocompromised personnel develop more } \\
\text { complications if infected }\end{array}$ & $\begin{array}{l}2100 \\
(99.2)\end{array}$ & $\begin{array}{c}6 \\
(0.3)\end{array}$ & $\begin{array}{c}11 \\
(0.5)\end{array}$ \\
\hline 5. & $\begin{array}{l}\text { COVID-19 virus is spread mainly through } \\
\text { respiratory droplets. }\end{array}$ & $\begin{array}{l}1956 \\
(92.4)\end{array}$ & $\begin{array}{l}56 \\
(2.6)\end{array}$ & $\begin{array}{l}105 \\
(5.0)\end{array}$ \\
\hline 6. & $\begin{array}{l}\text { Transmission of COVID-19 virus can only happen } \\
\text { when a person developed symptoms }\end{array}$ & $\begin{array}{r}1405 \\
(66.4) \\
\end{array}$ & $\begin{array}{c}474 \\
(22.4) \\
\end{array}$ & $\begin{array}{c}238 \\
(11.2)\end{array}$ \\
\hline 7. & COVID-19 virus strain can mutate over time. & $\begin{array}{c}1725 \\
(81.5)\end{array}$ & $\begin{array}{c}49 \\
(2.3)\end{array}$ & $\begin{array}{c}343 \\
(16.2)\end{array}$ \\
\hline
\end{tabular}

Multiple logistics regression was performed subsequently. Respondents within the 30-49 age group (vs. 18-29 age group, OR: 1.56, CI: 1.17-2.08, $p=0.002$ ), Chinese (vs. Malay, OR $=1.77, \mathrm{CI}: 1.16-2.70, p=0.008$ ), those with tertiary (vs. primary, $\mathrm{OR}=6.76$, CI: $1.46-31.27, p=0.015$ ) and medical education (vs. no medical education, $\mathrm{OR}=1.92$, CI: $1.34-2.75, p<0.001$ ), people with high household income (vs. low household income, OR: 2.82, CI: $1.53-5.19, p=0.001$ ) obtained higher knowledge scores. Meanwhile, patients of Indian ethnicity (vs. Malay, OR: 0.37, CI: $0.23-0.59, p<0.001$ ) scored significantly lower (Table 4). 
Table 3. Univariate logistic regression for significant factors associated with knowledge on COVID-19, knowledge on antibiotics resistance, practice and attitudes score $(n=2117)$.

\begin{tabular}{|c|c|c|c|c|c|c|c|c|}
\hline \multirow[t]{2}{*}{ Variable } & \multicolumn{2}{|c|}{$\begin{array}{c}\text { Knowledge on Antibiotics } \\
\text { Resistance }\end{array}$} & \multicolumn{2}{|c|}{ Knowledge on COVID-19 } & \multicolumn{2}{|c|}{ Practice Scores } & \multicolumn{2}{|c|}{ Attitude Scores } \\
\hline & $\begin{array}{l}\text { Crude OR } \\
(95 \% \text { CI })\end{array}$ & $p$ & $\begin{array}{l}\text { Crude OR } \\
(95 \% \text { CI })\end{array}$ & $p$ & $\begin{array}{l}\text { Crude OR } \\
(95 \% \text { CI })\end{array}$ & $p$ & $\begin{array}{l}\text { Crude OR } \\
(95 \% \text { CI })\end{array}$ & $p$ \\
\hline \multicolumn{9}{|l|}{ Age, years } \\
\hline $18-29$ & Reference & & Reference & & Reference & & Reference & \\
\hline $30-49$ & $1.43(1.17-1.75)$ & $<0.001$ & $1.88(1.46-2.42)$ & $<0.001$ & $1.79(1.29-2.48)$ & $<0.001$ & $1.29(0.93-1.80)$ & 0.128 \\
\hline$>50$ & $2.42(1.49-3.93)$ & $<0.001$ & $1.65(0.80-3.39)$ & 0.173 & $1.64(0.64-4.18)$ & 0.304 & $1.09(0.46-2.63)$ & 0.834 \\
\hline \multicolumn{9}{|l|}{ Gender } \\
\hline Male & Reference & & Reference & & Reference & & Reference & \\
\hline Female & $1.00(0.82-1.23)$ & 0.975 & $1.05(0.79-1.38)$ & 0.740 & $2.04(1.47-2.83)$ & $<0.001$ & $2.21(1.59-3.06)$ & $<0.001$ \\
\hline \multicolumn{9}{|l|}{ Ethnicity } \\
\hline Malay & Reference & & Reference & & Reference & & Reference & \\
\hline Chinese & $2.94(2.36-3.68)$ & $<0.001$ & $2.38(1.59-3.57)$ & $<0.001$ & $1.19(0.78-1.79)$ & 0.404 & $0.73(0.50-1.06)$ & 0.101 \\
\hline Indian & $1.50(1.01-2.23)$ & 0.044 & $0.48(0.31-0.74)$ & 0.001 & $2.07(0.83-5.18)$ & 0.119 & $0.84(0.43-1.67)$ & 0.625 \\
\hline Others & $0.84(0.59-1.20)$ & 0.330 & $1.13(0.72-1.77)$ & 0.590 & $1.49(0.79-2.83)$ & 0.214 & $1.58(0.78-3.18)$ & 0.201 \\
\hline \multicolumn{9}{|l|}{ Education } \\
\hline Primary or below & Reference & & Reference & & Reference & & Reference & \\
\hline Secondary & $0.55(0.06-4.76)$ & 0.583 & $\begin{array}{c}2.52 \\
(0.55-11.54)\end{array}$ & 0.233 & - & - & $\begin{array}{c}2.50 \\
(0.28-22.13)\end{array}$ & 0.410 \\
\hline Tertiary & $\begin{array}{c}3.32 \\
(0.39-27.63) \\
\end{array}$ & 0.267 & $\begin{array}{c}11.07 \\
(2.46-49.82)\end{array}$ & 0.002 & - & - & $\begin{array}{c}1.97 \\
(0.24-16.46) \\
\end{array}$ & 0.532 \\
\hline \multicolumn{9}{|l|}{ Occupation } \\
\hline Government & Reference & & Reference & & Reference & & Reference & \\
\hline Private & $0.44(0.36-0.54)$ & $<0.001$ & $0.55(0.39-0.75)$ & $<0.001$ & $0.87(0.60-1.26)$ & 0.465 & $0.49(0.33-0.44)$ & 0.001 \\
\hline Student & $0.39(0.26-0.58)$ & $<0.001$ & $0.48(0.29-0.79)$ & 0.004 & $0.37(0.22-0.61)$ & $<0.001$ & $0.44(0.24-0.81)$ & 0.009 \\
\hline Unemployed & $0.23(0.16-0.32)$ & $<0.001$ & $0.39(0.27-0.58)$ & $<0.001$ & $1.86(0.98-3.54)$ & 0.058 & $0.66(0.38-1.15)$ & 0.141 \\
\hline Retiree & $0.73(0.28-1.91)$ & 0.527 & $0.48(0.14-1.70)$ & 0.256 & - & - & $0.88(0.11-6.81)$ & 0.904 \\
\hline \multicolumn{9}{|l|}{$\begin{array}{l}\text { Healthcare related } \\
\text { education }\end{array}$} \\
\hline No & Reference & & Reference & & Reference & & Reference & \\
\hline Yes & $6.64(5.39-8.18)$ & $<0.001$ & $2.36(1.69-3.30)$ & $<0.001$ & $1.65(1.11-2.46)$ & 0.014 & $1.33(0.91-1.95)$ & 0.138 \\
\hline \multicolumn{9}{|l|}{ Chronic Disease } \\
\hline No & Reference & & Reference & & Reference & & Reference & \\
\hline Yes & $1.11(0.85-1.45)$ & 0.454 & $1.50(0.98-2.28)$ & 0.057 & $1.01(0.63-1.63)$ & 0.971 & $0.94(0.59-1.49)$ & 0.787 \\
\hline \multicolumn{9}{|l|}{ Household Income } \\
\hline$<\mathrm{RM} 4850$ & Reference & & Reference & & Reference & & Reference & \\
\hline $\begin{array}{l}\text { RM 4850-RM } \\
10,970\end{array}$ & $2.58(2.10-3.17)$ & $<0.001$ & $2.19(1.65-2.90)$ & $<0.001$ & $0.86(0.61-1.22)$ & 0.403 & $0.76(0.54-1.07)$ & 0.112 \\
\hline$\geq$ RM 10,971 & $3.55(2.69-4.67)$ & $<0.001$ & $4.95(2.78-8.82)$ & $<0.001$ & $0.73(0.46-1.15)$ & 0.174 & $1.05(0.62-1.77)$ & 0.862 \\
\hline \multicolumn{9}{|l|}{ Region } \\
\hline Central & Reference & & Reference & & Reference & & Reference & \\
\hline Northern & $1.63(1.30-2.04)$ & $<0.001$ & $0.92(0.67-1.27)$ & 0.602 & $1.29(0.85-1.94)$ & 0.234 & $1.15(0.75-1.75)$ & 0.524 \\
\hline Southern & $0.99(0.73-1.36)$ & 0.962 & $0.59(0.40-0.85)$ & 0.005 & $0.81(0.50-1.32)$ & 0.401 & $0.57(0.37-0.89)$ & 0.015 \\
\hline Eastern & $1.43(1.03-2.00)$ & 0.036 & $0.74(0.48-1.16)$ & 0.193 & $0.79(0.46-1.34)$ & 0.375 & $0.85(0.48-1.50)$ & 0.570 \\
\hline Borneo & $1.14(0.84-1.53)$ & 0.401 & $0.96(0.64-1.45)$ & 0.850 & $1.94(1.03-3.63)$ & 0.039 & $1.37(0.76-2.44)$ & 0.292 \\
\hline
\end{tabular}


Table 4. Multiple logistic regression for significant factors associated with knowledge on COVID-19, knowledge on antibiotics resistance, practice and attitudes score $(n=2117)$.

\begin{tabular}{|c|c|c|}
\hline Variable & \multicolumn{2}{|c|}{ Knowledge on COVID-19 } \\
\hline \multicolumn{3}{|l|}{ Age } \\
\hline $18-29$ years & Reference & \\
\hline $30-49$ years & $1.56(1.17-2.08)$ & 0.002 \\
\hline$>50$ year & $1.15(0.52-2.52)$ & 0.737 \\
\hline \multicolumn{3}{|l|}{ Ethnicity } \\
\hline Malay & Reference & \\
\hline Chinese & $1.77(1.16-2.70)$ & 0.008 \\
\hline Indian & $0.37(0.23-0.59)$ & $<0.001$ \\
\hline Others & $1.41(0.88-2.25)$ & 0.157 \\
\hline \multicolumn{3}{|l|}{ Education } \\
\hline \multicolumn{3}{|l|}{ Primary or below } \\
\hline Secondary & $2.08(0.44-9.74)$ & 0.353 \\
\hline Tertiary & $6.76(1.46-31.27)$ & 0.015 \\
\hline \multicolumn{3}{|c|}{ Healthcare related education } \\
\hline \multicolumn{3}{|l|}{ No } \\
\hline Yes & $1.92(1.34-2.75)$ & $<0.001$ \\
\hline \multicolumn{3}{|l|}{ Household Income } \\
\hline \multicolumn{3}{|l|}{$<\mathrm{RM} 4850$} \\
\hline RM 4850-RM 10,970 & $1.32(0.96-1.82)$ & 0.093 \\
\hline \multirow{3}{*}{$\geq \mathrm{RM} 10,971$} & $2.82(1.53-5.19)$ & 0.001 \\
\hline & \multicolumn{2}{|c|}{ Knowledge on antibiotics resistance } \\
\hline & Adjusted OR (95\%CI) & $p$ \\
\hline \multicolumn{3}{|l|}{ Ethnicity } \\
\hline Malay & Reference & \\
\hline Chinese & $2.36(1.82-3.05)$ & $<0.001$ \\
\hline Indian & $1.05(0.67-1.64)$ & 0.828 \\
\hline Others & $0.88(0.59-1.30)$ & 0.511 \\
\hline \multicolumn{3}{|l|}{ Occupation } \\
\hline Government & Reference & \\
\hline Private & $0.76(0.59-0.98)$ & 0.033 \\
\hline Student & $0.58(0.37-0.91)$ & 0.018 \\
\hline Unemployed & $0.66(0.45-0.97)$ & 0.034 \\
\hline Retiree & $0.74(0.25-2.17)$ & 0.586 \\
\hline \multicolumn{3}{|c|}{ Healthcare related education } \\
\hline No & Reference & \\
\hline Yes & $5.25(4.17-6.61)$ & $<0.001$ \\
\hline \multicolumn{3}{|l|}{ Household Income } \\
\hline$<\mathrm{RM} 4850$ & Reference & \\
\hline RM 4850-RM 10,970 & $1.68(1.32-2.14)$ & $<0.001$ \\
\hline$\geq \mathrm{RM} \mathrm{10,971}$ & $2.41(1.75-3.31)$ & $<0.001$ \\
\hline
\end{tabular}


Table 4. Cont.

\begin{tabular}{|c|c|c|}
\hline Variable & \multicolumn{2}{|c|}{ Knowledge on COVID-19 } \\
\hline & \multicolumn{2}{|l|}{ Practice scores } \\
\hline \multicolumn{3}{|l|}{ Age, years } \\
\hline $18-29$ & Reference & \\
\hline $30-49$ & $2.07(1.38-3.10)$ & $<0.001$ \\
\hline$>50$ & $1.87(0.63-5.57)$ & 0.262 \\
\hline \multicolumn{3}{|l|}{ Gender } \\
\hline Male & Reference & \\
\hline Female & $1.90(1.35-2.67)$ & $<0.001$ \\
\hline \multicolumn{3}{|l|}{ Medical education } \\
\hline No & Reference & \\
\hline Yes & $1.89(1.23-2.90)$ & 0.003 \\
\hline \multicolumn{3}{|l|}{ Household Income } \\
\hline \multicolumn{3}{|l|}{$<\mathrm{RM} 4850$} \\
\hline RM 4850-RM 10,970 & $0.60(0.41-0.89)$ & 0.013 \\
\hline$\geq$ RM 10,971 & $0.51(0.30-0.86)$ & 0.012 \\
\hline \multicolumn{3}{|c|}{ Attitude Scores } \\
\hline \multicolumn{3}{|l|}{ Gender } \\
\hline Male & Reference & \\
\hline Female & $2.12(1.51-2.99)$ & $<0.001$ \\
\hline \multicolumn{3}{|l|}{ Occupation } \\
\hline Government & Reference & \\
\hline Private & $0.49(0.32-0.75)$ & 0.001 \\
\hline Student & $0.42(0.22-0.79)$ & 0.008 \\
\hline Unemployed & $0.48(0.27-0.86)$ & 0.014 \\
\hline Retiree & $0.98(0.13-7.71)$ & 0.985 \\
\hline \multicolumn{3}{|l|}{ Household Income } \\
\hline$<\mathrm{RM} 4850$ & Reference & \\
\hline RM 4850-RM 10,970 & $0.59(0.41-0.86)$ & 0.006 \\
\hline$\geq \mathrm{RM} 10,971$ & $0.82(0.47-1.41)$ & 0.472 \\
\hline \multicolumn{3}{|l|}{ Region } \\
\hline \multicolumn{3}{|l|}{ Central } \\
\hline Northern & $1.01(0.65-1.56)$ & 0.970 \\
\hline Sothern & $0.55(0.35-0.88)$ & 0.012 \\
\hline Eastern & $0.77(0.43-1.38)$ & 0.381 \\
\hline Borneo & $1.17(0.64-2.11)$ & 0.613 \\
\hline
\end{tabular}

Notes: OR = odds ratio, $\mathrm{CI}$ = confidence interval; Backward stepwise multiple logistic regression analysis. Multicollinearity and interaction term were checked and not found. The Hosmer-Lemeshow test, Nagelkerke classification table and area under the curve were applied to check model fitness. Knowledge on Covid-19: Hosmer-Lemeshow test: 0.579, Nagelkerke: 0.139, Area under the curve: 86.6; Knowledge on antibiotics resistance: Hosmer-Lemeshow test: <0.001; Nagelkerke test: 0.294; Area under the curve: 77.1; Attitude: HosmerLemeshow test: 0.290, Nagelkerke test: 0.055, Area under the curve: 92.3; Practice: Hosmer-Lemeshow test: 0.991; Nagelkerke test: 0.068; Area under the curve: 92.3. 


\subsection{Knowledge on Antibiotics Use and Resistance}

Respondents were required to answer ten questions regarding antibiotics use and antibiotics resistance. The mean score of the respondents was $6.12(\mathrm{SD}=2.34$, range $0-10)$, giving $61.2 \%$ overall proportion of correct answers $(6.12 / 10 \times 100)$. Majority of the respondents were not able to obtain a score of 8 or more, indicating poor knowledge towards antibiotics resistance (1430, 67.5\%). $916(43.3 \%)$ respondents falsely believed that taking antibiotics could speed up the recovery process of all infections. Less than half of the respondents $(1037,49.0 \%)$ were aware that antibiotics are effective against bacterial infection only. More than two-fifths of the respondents $(876,41.4 \%)$ were unsure whether antibiotics resistance would cause mortality (Table 5). Differences in knowledge scores on antibiotics resistance were significantly different across all demographic characteristics except gender, educational level and chronic disease status (Table 3).

Table 5. Knowledge on antibiotics use and resistance $(n=2117)$.

\begin{tabular}{|c|c|c|c|c|}
\hline No. & Statement & Correct & Incorrect & Unsure \\
\hline 1. & Bacteria strains can mutate rapidly over time & $\begin{array}{l}1466 \\
(69.3)\end{array}$ & $\begin{array}{l}166 \\
(7.8)\end{array}$ & $\begin{array}{c}485 \\
(22.9)\end{array}$ \\
\hline 2. & $\begin{array}{l}\text { Development of new antimicrobials/vaccinations } \\
\text { is simple and does not take up much time. }\end{array}$ & $\begin{array}{c}1754 \\
(82.9)\end{array}$ & $\begin{array}{c}174 \\
(8.2)\end{array}$ & $\begin{array}{l}189 \\
(8.9)\end{array}$ \\
\hline 3. & Taking antibiotic can prevent all infection & $\begin{array}{c}1369 \\
(64.7)\end{array}$ & $\begin{array}{c}368 \\
(17.4)\end{array}$ & $\begin{array}{c}380 \\
(17.9)\end{array}$ \\
\hline 4. & $\begin{array}{l}\text { Taking antibiotic can speed up the recovery } \\
\text { process of all infection }\end{array}$ & $\begin{array}{c}779 \\
(36.8)\end{array}$ & $\begin{array}{c}916 \\
(43.3)\end{array}$ & $\begin{array}{c}422 \\
(19.9)\end{array}$ \\
\hline 5. & $\begin{array}{l}\text { Antibiotic dosage dose adjustment can be done } \\
\text { without consultation from the professional } \\
\text { medical practitioners }\end{array}$ & $\begin{array}{c}1962 \\
(92.6)\end{array}$ & $\begin{array}{c}50 \\
(2.4)\end{array}$ & $\begin{array}{c}105 \\
(5.0)\end{array}$ \\
\hline 6. & $\begin{array}{l}\text { Antibiotics is effective against bacterial } \\
\text { infection only }\end{array}$ & $\begin{array}{c}1037 \\
(49.0)\end{array}$ & $\begin{array}{c}540 \\
(25.5)\end{array}$ & $\begin{array}{c}540 \\
(25.5)\end{array}$ \\
\hline 7. & Antibiotic resistance can cause mortality & $\begin{array}{c}1241 \\
(58.6)\end{array}$ & $\begin{array}{c}110 \\
(5.2)\end{array}$ & $\begin{array}{c}766 \\
(36.2)\end{array}$ \\
\hline 8. & $\begin{array}{l}\text { Like COVID-19, resistant bacteria strain can cause } \\
\text { similar pandemic events }\end{array}$ & $\begin{array}{l}1089 \\
(51.4)\end{array}$ & $\begin{array}{c}181 \\
(8.6)\end{array}$ & $\begin{array}{c}847 \\
(40.0)\end{array}$ \\
\hline 9. & $\begin{array}{l}\text { Misuse of antibiotics will accelerate the antibiotic } \\
\text { resistance process }\end{array}$ & $\begin{array}{c}1261 \\
(59.6) \\
\end{array}$ & $\begin{array}{l}145 \\
(6.8) \\
\end{array}$ & $\begin{array}{c}711 \\
(33.6)\end{array}$ \\
\hline 10. & $\begin{array}{l}\text { Hand hygiene is essential to prevent } \\
\text { antibiotic resistance. }\end{array}$ & $\begin{array}{c}1001 \\
(47.3)\end{array}$ & $\begin{array}{c}512 \\
(24.2)\end{array}$ & $\begin{array}{c}604 \\
(28.5)\end{array}$ \\
\hline
\end{tabular}

The following characteristics predicted higher knowledge score on antibiotics use and resistance: Chinese respondents (vs. Malay, OR:2.36, CI: 1.82-3.05, $p<0.001$ ), medical education (vs. no medical education, OR: 5.25, CI: 4.17-6.61, $p<0.001$ ), middle household income (vs. low household income, OR:1.68, CI:1.32-2.14, $p<0.001$ ), high household income (vs. low household income, OR: 2.41, CI: 1.75-3.31, $p<0.001$ ). In contrast, those worked in the private sectors (vs. government, OR: 0.76, CI: 0.59-0.98, $p=0.033$ ), students (vs. government, OR: 0.58, CI: 0.37-0.91, $p=0.018$ ) and unemployed (vs. government, OR: 0.66 , CI: $0.45-0.97, p=0.034$ ) scored significantly lower in this domain (Table 4 ).

\subsection{Practice of Preventive Measures}

The practices of preventive measures during COVID-19 were measured using 11 questions. The mean practice score was $10.42(\mathrm{SD}=1.26$, range: $0-11)$, giving an overall $94.7 \%$ of good practices $(10.42 / 11 \times 100)$. Majority of the respondents $(1953,92.3 \%)$ practiced at least 9 preventive measures during the COVID-19 pandemic. The two preventive measures that 
were least practiced were hand washing for at least $20 \mathrm{~s}(1796,84.8 \%)$ and hand washing before touching face $(1861,87.9 \%$ ) (Table 6).

Table 6. Practice of preventive measures during the coronavirus disease 2019 (COVID-19) pandemic $(n=2117)$.

\begin{tabular}{|c|c|c|c|}
\hline No. & Statement & Yes & No \\
\hline 1. & $\begin{array}{l}\text { Frequent hand washing after in contact with frequent } \\
\text { touched surfaces. }\end{array}$ & $\begin{array}{c}2028 \\
(95.8)\end{array}$ & $\begin{array}{c}89 \\
(4.2)\end{array}$ \\
\hline 2. & Wash hand before and after touching eyes, nose and mouth & $\begin{array}{c}1861 \\
(87.9)\end{array}$ & $\begin{array}{c}256 \\
(12.1)\end{array}$ \\
\hline 3. & Wash hand with water and soap or sanitizer & $\begin{array}{c}2093 \\
(98.9)\end{array}$ & $\begin{array}{c}24 \\
(1.1)\end{array}$ \\
\hline 4. & Wash hand for at least $20 \mathrm{~s}$ & $\begin{array}{c}1796 \\
(84.8)\end{array}$ & $\begin{array}{c}321 \\
(15.2)\end{array}$ \\
\hline 5. & Wear face mask in public area & $\begin{array}{r}2107 \\
(99.5)\end{array}$ & $\begin{array}{c}10 \\
(0.5)\end{array}$ \\
\hline 6. & Close mouth and nose when sneezing or coughing & $\begin{array}{c}2097 \\
(99.1)\end{array}$ & $\begin{array}{c}20 \\
(0.9)\end{array}$ \\
\hline 7. & Always bring along sanitizer or wet wipes & $\begin{array}{c}1942 \\
(91.7)\end{array}$ & $\begin{array}{r}175 \\
(8.3)\end{array}$ \\
\hline 8. & $\begin{array}{l}\text { Always maintain physical distancing at least } 1 \mathrm{~m} \\
\text { from others }\end{array}$ & $\begin{array}{c}2034 \\
(96.1)\end{array}$ & $\begin{array}{c}83 \\
(3.9)\end{array}$ \\
\hline 9. & Avoid crowded and narrow places & $\begin{array}{l}2048 \\
(96.7)\end{array}$ & $\begin{array}{c}69 \\
(3.3)\end{array}$ \\
\hline 10. & Avoid chatting and speaking at close distance & $\begin{array}{c}2005 \\
(94.7)\end{array}$ & $\begin{array}{r}112 \\
(5.3)\end{array}$ \\
\hline 11. & $\begin{array}{l}\text { Limit physical contact: no handshake policy, greeting with } \\
\text { hand on the chest. }\end{array}$ & $\begin{array}{c}2041 \\
(96.4)\end{array}$ & $\begin{array}{c}76 \\
(3.6)\end{array}$ \\
\hline
\end{tabular}

The practice score was associated with age, gender, occupation, healthcare related education background and region of residence (Table 3). Females (vs. male, OR: 1.90, CI: 1.35-2.67 $p<0.001$ ), aged between 30-49 (vs. age 18-29, OR: 2.07, CI: 1.38-3.11, $p<0.001$ ), those with medical education (vs. no medical education, OR: 1.89, CI: 1.23-2.90, $p=0.003$ ) possessed higher practice score. Interestingly, those with middle income (vs. lower income, OR: 0.60, CI: $0.41-0.89, p=0.013$ ) and higher income (vs. lower income, OR: $0.51, \mathrm{CI}$ : $0.30-0.86, p=0.012$ ) obtained lower practice scores (Table 4 ).

\subsection{Attitude towards New Norm}

Seven questions were asked to determine the attitude of the respondents towards the new norms during the COVID-19 pandemic. The mean score was 6.49 (SD $=0.83$, Range: $0-7)$, indicating an overall $92.7 \%$ of positive attitude among the respondents $(6.49 / 7 \times 100)$. Majority of the respondents strongly agreed that quarantine should be made mandatory for all arrival from overseas $(2058,97.2 \%)$ and wearing face masks should be made mandatory in all public areas $(1989,94.0 \%)$. However, more than a quarter of the respondents $(648,30.6 \%)$ remained neutral or disagreed that working from home is productive (Table 7). Gender, occupation and region of residence were found to be associated with attitude scores (Table 3). Female respondents (vs. male, OR: 2.12, CI: $1.51-2.99, p<0.001$ ) were more likely to have a higher attitude scores, while students (vs. government, OR: 0.42 , CI: $0.22-0.79, p=0.008$ ), unemployed people (vs. government, OR: 0.48 , CI: $0.27-0.86, p=0.014$ ), people working in the private sectors (vs. government, OR: 0.49 , CI: $0.32-0.75, p=0.001$ ), those with middle household income (vs. low income, OR: 
0.59, CI: $0.41-0.86, p=0.006)$, people resided in the Southern region (vs. Central region, OR: 0.55, CI: $0.35-0.88, p=0.012$ ) scored significantly lower (Table 4).

Table 7. Attitude towards new norm during the COVID-19 pandemic $(n=2117)$.

\begin{tabular}{|c|c|c|c|c|c|c|}
\hline No. & Statement & $\begin{array}{l}\text { Strongly } \\
\text { Disagree }\end{array}$ & Disagree & Neutral & Agree & $\begin{array}{l}\text { Strongly } \\
\text { Agree }\end{array}$ \\
\hline 1. & $\begin{array}{l}\text { Body temperature } \\
\text { monitoring should be } \\
\text { practiced at all public areas }\end{array}$ & $\begin{array}{c}19 \\
(0.9)\end{array}$ & $\begin{array}{c}15 \\
(0.7)\end{array}$ & $\begin{array}{c}84 \\
(4.0)\end{array}$ & $\begin{array}{c}238 \\
(11.2)\end{array}$ & $\begin{array}{l}1761 \\
(83.2)\end{array}$ \\
\hline 2. & $\begin{array}{l}\text { Availability of hand sanitizer } \\
\text { in public area will encourage } \\
\text { frequent hand cleaning }\end{array}$ & $\begin{array}{c}11 \\
(0.5)\end{array}$ & $\begin{array}{c}9 \\
(0.4)\end{array}$ & $\begin{array}{c}36 \\
(1.7)\end{array}$ & $\begin{array}{l}149 \\
(7.0)\end{array}$ & $\begin{array}{l}1912 \\
(90.4)\end{array}$ \\
\hline 3. & $\begin{array}{l}\text { Face mask wearing should be } \\
\text { made mandatory in all } \\
\text { public area }\end{array}$ & $\begin{array}{c}12 \\
(0.6)\end{array}$ & $\begin{array}{c}7 \\
(0.3)\end{array}$ & $18(0.9)$ & $\begin{array}{c}91 \\
(4.3)\end{array}$ & $\begin{array}{l}1989 \\
(93.9)\end{array}$ \\
\hline 4. & $\begin{array}{l}\text { Work from home is } \\
\text { productive and should } \\
\text { be encouraged }\end{array}$ & $\begin{array}{c}61 \\
(2.9)\end{array}$ & $\begin{array}{c}81 \\
(3.8)\end{array}$ & $\begin{array}{c}506 \\
(23.9)\end{array}$ & $\begin{array}{c}433 \\
(20.5)\end{array}$ & $\begin{array}{l}1036 \\
(48.9)\end{array}$ \\
\hline 5. & $\begin{array}{l}\text { Table distancing at restaurant } \\
\text { should be continued }\end{array}$ & $\begin{array}{c}14 \\
(0.7)\end{array}$ & $\begin{array}{c}8 \\
(0.4)\end{array}$ & $\begin{array}{c}68 \\
(3.2)\end{array}$ & $\begin{array}{c}229 \\
(10.8)\end{array}$ & $\begin{array}{l}1798 \\
(84.9)\end{array}$ \\
\hline 6. & $\begin{array}{l}\text { Quarantine should be made } \\
\text { mandatory for all arrival } \\
\text { from overseas }\end{array}$ & $\begin{array}{c}9 \\
(0.4)\end{array}$ & $\begin{array}{c}6 \\
(0.3)\end{array}$ & $\begin{array}{c}12 \\
(0.6)\end{array}$ & $\begin{array}{c}32 \\
(1.5)\end{array}$ & $\begin{array}{c}2058 \\
(97.2)\end{array}$ \\
\hline 7. & $\begin{array}{l}\text { Continuous education from } \\
\text { the government had helped } \\
\text { me to face this } \\
\text { pandemic better }\end{array}$ & $\begin{array}{c}21 \\
(1.0)\end{array}$ & $\begin{array}{c}10 \\
(0.5)\end{array}$ & $\begin{array}{c}75 \\
(3.5)\end{array}$ & $\begin{array}{c}177 \\
(8.4)\end{array}$ & $\begin{array}{c}1834 \\
(86.6)\end{array}$ \\
\hline
\end{tabular}

\subsection{Correlations between Different Domains}

The knowledge scores on COVID-19 had a significant and moderate correlation with the knowledge scores on antibiotics $(r=0.444, p<0.001)$, and a weak but significant correlation with the practice scores $(\mathrm{r}=0.076, p<0.001)$ and attitudes scores $(\mathrm{r}=0.117$, $p<0.001)$. Attitude scores demonstrated a significant but weak correlation with the practice scores $(\mathrm{r}=0.187, p<0.001)$ (Table 8$)$.

Table 8. Correlation matrix (Spearman) of knowledge on COVID-19, knowledge on antibiotics, attitude, practice scores.

\begin{tabular}{ccccc}
\hline Correlations & $\begin{array}{c}\text { Knowledge on } \\
\text { Covid-19 Scores }\end{array}$ & $\begin{array}{c}\text { Knowledge on } \\
\text { Antibiotics Scores }\end{array}$ & $\begin{array}{c}\text { Practice } \\
\text { Scores }\end{array}$ & $\begin{array}{c}\text { Attitude } \\
\text { Scores }\end{array}$ \\
\hline $\begin{array}{c}\text { Knowledge on } \\
\text { Covid-19 scores }\end{array}$ & 1 & - & - & - \\
\hline $\begin{array}{c}\text { Knowledge on } \\
\text { antibiotics scores }\end{array}$ & $0.444^{*}$ & 1 & - & - \\
\hline Practice scores & $0.076^{*}$ & $0.026(p=0.240)$ & 1 & - \\
\hline Attitude scores & $0.117^{*}$ & $0.012(p=0.580)$ & $0.187^{*}$ & 1 \\
\hline$p<0.001$. & & &
\end{tabular}

\section{Discussion}

A large number of COVID-19 related KAP studies were conducted during the pandemic, as it is important to gauge the effectiveness of public educational intervention by the health authorities. A timely update of the scenario at the ground is important to inform policymakers on the current gap of knowledge among the public. From this study, it was 
found that the general population of Malaysia had good knowledge towards COVID19, practiced appropriate preventive measures to prevent COVID-19 and demonstrated positive attitudes towards the new norm during the pandemic. However, knowledge on antibiotics use and resistance was poor among the public.

The knowledge of the general public in Malaysia with regards to COVID-19 was high, with a mean score of 6.36 and an overall correct rate of $91 \%$. Most of the KAP studies conducted previously reported adequate knowledge of the respondents towards COVID-19 [10-12]. This was consistent with a previous local study, where the overall correct responses were more than $80 \%$ [19]. However, there was a notable uncertainty regarding the asymptomatic transmission of COVID-19. The evidence of asymptomatic transmission remained debatable, as some studies suggesting lower rate of transmission from an asymptomatic patient $[25,26]$. This may have contributed to the difficulties among the respondents in obtaining accurate information.

In congruence with other studies, we found that respondents of Chinese ethnicity, middle-aged adults, those with tertiary education and people with higher household income were more knowledgeable regarding COVID-19 [11,12,19]. Not surprisingly, respondents with tertiary education were 6.7 times more likely to possess good knowledge towards COVID-19, which might be attributed to their higher health literacy. While it was evident that COVID-19 patients with pre-existing comorbidities had higher risk of mortality [27], surprisingly, the knowledge among people with chronic diseases did not differ significantly from healthy individuals. This suggested that both groups of respondents had gained COVID-19 related information over time through regular mass education campaigns.

Overall, the respondents had low antibiotics-related knowledge with a mean score of 6.1 out of 10 . Notably, more than two-fifths (43\%) of the respondents believed that taking antibiotics can speed up the recovery process of all infections. This was comparable to a large-scale local study, where $63 \%$ of the population believed the same [23]. Additionally, less than half (49\%) of our respondents knew that antibiotics are only effective against bacteria. Similarly, previous studies reported that majority of the population believed viral infection could be cured by taking antibiotics [22,23]. The poor antibiotics-related knowledge among our respondents suggests increased risk of antibiotics misuse, which may further exacerbate global antibiotics resistance [28,29].

Respondents with higher household income had significantly better knowledge regarding antibiotics. This was in line with previous studies [30,31], as people from higher social classes were more well equipped with facilities to access health-related information. Notably, respondents with a medical-related educational background were 5 times more likely to have good knowledge score pertaining to antibiotics use and resistance $[30,32,33]$. This is because antibiotics-related syllabuses were commonly incorporated in training programs for healthcare providers.

Overall, the public had better knowledge towards COVID-19 than antibiotics use. Notably, information regarding COVID-19 was constantly disseminated among the general public and relevant rules were being enforced concurrently. Meanwhile, antibiotics knowledge was disseminated only through antibiotics awareness campaigns occasionally, of which the public may not aware. As there were no penalties implemented, the general public was not obliged to abide any rules of prudent antibiotics use. This may explain the higher level of knowledge towards COVID-19 in comparison with antibiotics use [7,19].

Majority of the Malaysian public demonstrated good practice of COVID-19 preventive measures. In comparison to the previous local study conducted before the MCO, practices of frequent hand washing and avoiding crowded places had increased from $80 \%$ to more than $90 \%$ over time [19]. However, $15 \%$ of the respondents did not wash their hands for more than $20 \mathrm{~s}$, which was also reported in other studies [13,30].

It is noteworthy to highlight the high proportion in the practice of wearing face masks (99.5\%) in contrast with other studies. Previous studies show that a large proportion of the general public did not consider wearing a face mask as a preventive measure [12,18]. Azlan and colleagues reported that only $51.2 \%$ of Malaysians wore a face mask in public areas 
one week before the MCO [19]. This may be attributed to the shortage of face mask supply and mixed evidence on the effectiveness of face masks in preventing COVID-19 in the early stage of the outbreak [34]. Health authorities' messages, augmented with emergence of new evidence on the importance of face masks, may have changed the practice over time $[9,35,36]$.

Females and middle-aged adults had higher practice scores and were more likely to practice preventive measures. This was in concordance with previous studies in China and Vietnam, where male respondents were less compliant to the practice of COVID-19 preventive measures $[11,13]$. Older adults were twice as likely to have good practice compared to younger adults (18-29 years old), as they may perceive the higher risk of mortality if infected by COVID-19 [37].

It is worth mentioning that higher household income was associated with lower practice scores, consistent with the findings by Azlan et al. [19]. The impact of income lost and healthcare cost incurred once diagnosed with COVID-19 may be higher among those individuals with lower income, as some relied on daily wages and temporary works $[38,39]$. Hence, the lower income group had an unexpected higher compliance towards COVID-19 preventive measures.

The Malaysian population, specifically females and civil servants, had positive attitudes towards most of the new norms being implemented in the country. Majority of the respondents also agreed that continuous education from the government had prepared them to face the pandemic. The Director General of Health office and the Malaysian National Security Council held daily press statements and regular press conferences regarding COVID-19 since the outbreak began [40,41]. The public were informed regarding COVID19 daily statistics, new clusters information, preventive measures and implementation of new rules for COVID-19 prevention.

However, more than a quarter of the respondents were undecided regarding the productiveness of work from home measures. A previous survey in Malaysia reported similar findings, where $31 \%$ of the employees did not wish to continue working from home after the MCO and the productivity was affected by communication barriers and network issues [42]. Furthermore, vulnerable groups such as high school dropouts, informal workers and low-income households may involve jobs that cannot be performed from home. Therefore, the government plays a crucial role in reducing inequalities related to work from home opportunities, by enhancing human capital training and providing adequate support in technology facilities.

Notably, respondents working in the government sector possessed a higher knowledge score towards antibiotics and adopted a more positive attitude towards the new norm. This was similar to the finding by a local study conducted in the early of March 2020 [19]. This might be attributed to the high level of health literacy among the Malaysian civil servants [43]. Additionally, the civil servants also receive regular and first-hand information and directives from the government, which may further consolidate their knowledge and foster positive attitudes.

Based on our findings, the respondents' knowledge scores on COVID-19 were significantly correlated with the practice scores and attitudes scores. Attitude scores also demonstrated a significant correlation with the practice scores. Therefore, this was consistent with the KAP framework, where good knowledge and attitudes was associated with good practice among the public. However, good knowledge scores on antibiotics were not significantly correlated with good practice scores. Hence, this suggested that the current model of public educational strategies targeted on COVID-19 knowledge may be adequate to promote prudent practice of preventive measures among the general public.

\section{Strength and Limitations}

To our best knowledge, this was one of the first nationwide studies which assessed the knowledge of the general public towards antibiotics use and resistance after the COVID-19 outbreak, which was particularly important in the antimicrobial resistance era. The practice 
and attitude items covered in this study was more comprehensive than previous studies conducted in the earlier stage of COVID-19 outbreak [11,19]. We employed convenience and snowball sampling in this study through social media and the researchers' network, hence the response rate could not be estimated. The limitation of cross-sectional study design was that the temporal relationship of outcome and exposure could not be determined. Due to the online nature of this survey, there was an under-representation of male respondents, people with primary education, those above the age of 50, and working in the private sectors, in comparison to the actual demographic distribution of Malaysian population. Therefore, our findings might not be generalized to these populations. While we asked the age of respondents before their participation, we could not ensure that no people under 18 years of age participated due to the web-based nature of the questionnaire. Future studies should employ systematic stratified sampling to ensure generalizability of the findings.

\section{Conclusions}

The Malaysian general public had good knowledge, appropriate practice and positive attitudes towards COVID-19, suggesting the effectiveness of the current educational programs provided by the health authorities. However, the majority did not have adequate knowledge regarding antibiotics use and resistance. Incorporation of antibiotics-related information in public awareness programs is warranted, especially towards the population from a lower socioeconomic class.

Author Contributions: Conceptualization, all authors; methodology, all authors; formal analysis, C.T.C., T.Y.N., A.A.S. and K.S.T.; investigation, all authors; data curation, all authors; writingoriginal draft preparation, C.T.C.; writing-review and editing, M.L., J.C.Y.L., N.C.T.L., T.Y.N., A.A.S. and K.S.T.; supervision, K.S.T.; project administration, C.T.C., M.L. and J.C.Y.L.; funding acquisition, A.A.S. and K.S.T. All authors have read and agreed to the published version of the manuscript.

Funding: This research did not receive any specific grant from funding agencies in the public, commercial, or not-for-profit sectors.

Institutional Review Board Statement: The study was conducted according to the guidelines of the Declaration of Helsinki, and approved by the Institutional Review Board (or Ethics Committee) of NMRR (NMRR Registration Number: NMRR-20-1336-55250, Ethical approval number: KKM/NIHHSEC/P20-1463 (3) and date of approval: 16 July 2020.

Informed Consent Statement: Informed consent was obtained from all subjects involved in the study. Online informed consent has been obtained from the patient(s) to publish this paper.

Data Availability Statement: The dataset used in this study can be obtained from the authors upon reasonable requests.

Acknowledgments: We would like to thank the Director General of Health for his permission to publish this study. We would like to convey our appreciation to Narwani Hussin and Steven Lim Chee Loon in reviewing the content of the manuscript. We would also like to thank the Perak State Deputy Director of Health, Pn Zaiton binti Kamarruddin and Philip Rajan, Head of Clinical Research Centre Hospital Raja Permaisuri Bainun for their administrative support of this work. Special thanks to the Corporate Communication Unit, Ministry of Health Malaysia for their assistance to disseminate the questionnaire.

Conflicts of Interest: The authors declare no conflict of interest.

\section{Appendix A}

Survey tool

Please $\sqrt{ }$ at your response

Section A: Demographic Characteristics 


\begin{tabular}{|c|c|}
\hline Years: & Postcode: \\
\hline $\begin{array}{l}\text { Gender: } \\
\text { - } \quad \text { Male } \\
\text { - } \quad \text { Female }\end{array}$ & $\begin{array}{l}\text { Are you a healthcare provider (e.g., doctor, nurse) or } \\
\text { (bio)medical student? } \\
\text { - Yes } \\
\text { - } \quad \text { No }\end{array}$ \\
\hline $\begin{array}{l}\text { Race: } \\
\text { - } \quad \text { Malay } \\
\text { - } \quad \text { Chinese } \\
\text { - } \quad \text { Indian } \\
\text { - } \quad \text { Others }\end{array}$ & $\begin{array}{l}\text { Do you suffer from any chronic illness or poor } \\
\text { medical condition? (e.g., respiratory disease, heart } \\
\text { disease, metabolic disorders such as diabetes, } \\
\text { (previous) cancer treatment or other } \\
\text { diseases requiring chronic medication) } \\
\text { - Yes } \\
\text { - No }\end{array}$ \\
\hline $\begin{array}{l}\text { Highest Academic achievement: } \\
\text { - } \quad \text { Primary level } \\
\text { - } \quad \text { Secondary level } \\
\text { - } \quad \text { Tertiary level }\end{array}$ & $\begin{array}{l}\text { How did you know about this survey? } \\
\text { - } \quad \text { Social media (Facebook, Twitter, Instagram etc.) } \\
\text { - } \quad \text { Instant messaging (WhatsApp) } \\
\text { - } \quad \text { Email } \\
\text { - } \quad \text { Others }\end{array}$ \\
\hline
\end{tabular}

Are you currently employed?

- Full time

- Part-time

- Unemployed

- $\quad$ Retiree (private)

- Government pensioner
Monthly household income:

- $\quad<$ RM 4850

- $\quad$ RM 4501-RM 10,959

- $\quad>$ RM 10,959

Section B: Background Knowledge about COVID-19 Pandemic

\begin{tabular}{lllll}
\hline No. & \multicolumn{1}{c}{ Statements } & True & False & Unsure \\
\hline - & The COVID-19 pandemic is virus origin & & \\
\hline - & $\begin{array}{l}\text { The main clinical symptoms of COVID-19 are fever, } \\
\text { cough, sore throat and difficulty breathing }\end{array}$ & & \\
\hline
\end{tabular}

- COVID-19 can affect anyone at any stage of life

Elderly, child, people with co-morbidities and

- $\quad$ immunocompromised personnel are more susceptible to COVID-19 and develops more complications if infected

- COVID-19 virus is spread mainly through

respiratory droplets

- Transmission of COVID-19 virus can only happen when a

person developed symptoms

- $\quad$ COVID-19 virus strains can mutate over time

Section C: Preventive Measures during COVID-19 pandemic

Please indicate which preventive measure would you continue after the Covid-19 pandemic Hand Hygiene

\begin{tabular}{|c|c|c|c|}
\hline No. & Preventive Measures & Yes & No \\
\hline • & Frequent hand washing after in contact with frequent touched surfaces & & \\
\hline • & Wash your hands before and after touching eyes, nose and mouth & & \\
\hline$\bullet$ & 6 steps hand washing that lasts for at least $20 \mathrm{~s}$ & & \\
\hline
\end{tabular}




\begin{tabular}{|c|c|c|c|}
\hline No. & Preventive Measures & Yes & No \\
\hline • & Face mask wearing at crowded area, public transport & & \\
\hline • & Cough and sneeze etiquette & & \\
\hline$\bullet$ & Bringing along hand sanitizers or wipes whenever going out & & \\
\hline \multicolumn{4}{|c|}{ Social Interaction } \\
\hline No. & Preventive Measures & Yes & No \\
\hline • & Physical distancing at least $1 \mathrm{~m}$ & & \\
\hline • & Avoid crowded places & & \\
\hline$\bullet$ & Avoid talking in close distance & & \\
\hline - & Limit physical contact: no handshake policy, salam letak tangan di dada & & \\
\hline
\end{tabular}

Section D: Knowledge towards Antibiotic Use and Antibiotic Resistance

\begin{tabular}{|c|c|c|c|}
\hline No. & Statement & Yes & Unsure \\
\hline \multicolumn{4}{|c|}{ General knowledge } \\
\hline • & Bacteria strains can mutate rapidly in a short period of time & & \\
\hline$\bullet$ & $\begin{array}{l}\text { Developments of new antimicrobials/vaccinations is } \\
\text { simple and does not take up much time }\end{array}$ & & \\
\hline \multicolumn{4}{|c|}{ Antibiotics use } \\
\hline - & Antibiotic use can prevent all infections from getting worst & & \\
\hline$\bullet$ & Antibiotic use can help to fasten the recovery process & & \\
\hline$\bullet$ & $\begin{array}{l}\text { Antibiotic dosage adjustment can be done based on severity } \\
\text { of disease without seeking professional medical advice }\end{array}$ & & \\
\hline$\bullet$ & Antibiotic is used for bacterial infection only & & \\
\hline \multicolumn{4}{|c|}{ Antibiotics resistance } \\
\hline$\bullet$ & Antibiotic resistance can cause death & & \\
\hline$\bullet$ & $\begin{array}{l}\text { Like COVID-19, a new resistant bacteria strain can cause } \\
\text { similar or worst pandemic events }\end{array}$ & & \\
\hline & $\begin{array}{l}\text { Misuse of antibiotics will accelerate the antibiotic } \\
\text { resistance process }\end{array}$ & & \\
\hline$\bullet$ & $\begin{array}{l}\text { Hand hygiene practice is essential to prevent } \\
\text { antibiotic resistance }\end{array}$ & & \\
\hline
\end{tabular}

Section E: Adapting to a new norm Post COVID-19 pandemic

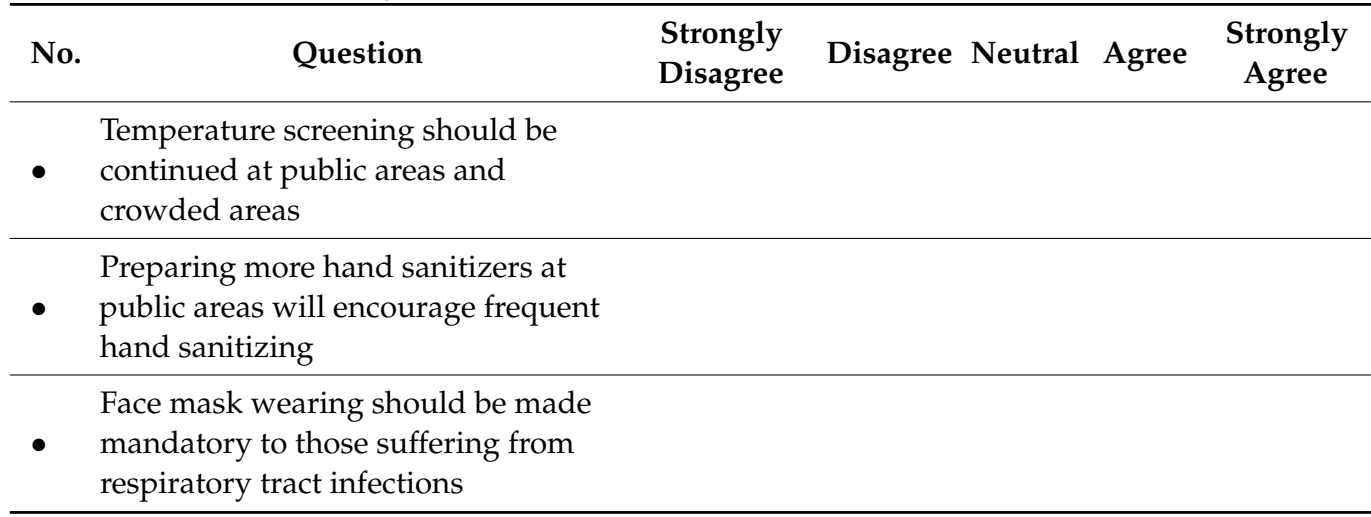




\begin{tabular}{|c|c|c|c|c|c|}
\hline No. & Question & $\begin{array}{l}\text { Strongly } \\
\text { Disagree }\end{array}$ & Disagree Neutral & Agree & $\begin{array}{l}\text { Strongly } \\
\text { Agree }\end{array}$ \\
\hline - & $\begin{array}{l}\text { Working from home is productive } \\
\text { and should be encouraged }\end{array}$ & & & & \\
\hline - & $\begin{array}{l}\text { Table distancing should be continued } \\
\text { at food outlets }\end{array}$ & & & & \\
\hline • & $\begin{array}{l}\text { Home quarantine should be made } \\
\text { compulsory to all } \\
\text { international travelers }\end{array}$ & & & & \\
\hline • & $\begin{array}{l}\text { Continuous education on infectious } \\
\text { disease by the government to public } \\
\text { is essential to prevent a new outbreak }\end{array}$ & & & & \\
\hline
\end{tabular}

\section{Appendix B}

Table A1. Comparison of demographic characteristics with knowledge, practice and attitudes score $(n=2117)$.

\begin{tabular}{|c|c|c|c|c|c|c|c|c|c|}
\hline \multirow[t]{2}{*}{ Variable } & \multirow[t]{2}{*}{ Freq $(n)$} & \multicolumn{2}{|c|}{$\begin{array}{l}\text { Knowledge on } \\
\text { COVID-19 }\end{array}$} & \multicolumn{2}{|c|}{$\begin{array}{l}\text { Knowledge on Antibiotics } \\
\text { Resistance }\end{array}$} & \multicolumn{2}{|c|}{$\begin{array}{c}\text { Practice of Preventive } \\
\text { Measures }\end{array}$} & \multicolumn{2}{|c|}{$\begin{array}{l}\text { Attitude towards New } \\
\text { Norm }\end{array}$} \\
\hline & & Mean (SD) & $p$-Value & Mean (SD) & $p$-Value & Mean (SD) & $p$-Value & Mean (SD) & $p$-Value \\
\hline \multicolumn{10}{|l|}{ Gender } \\
\hline Male & 571 & $6.27(0.97)$ & 0.002 & $6.12(2.30)$ & 0.995 & $10.2(1.57)$ & $<0.001$ & $6.34(1.05)$ & $<0.001$ \\
\hline Female & 1546 & $6.40(0.83)$ & & $6.12(2.35)$ & & $10.5(1.10)$ & & $6.55(0.72)$ & \\
\hline \multicolumn{10}{|l|}{ Age } \\
\hline $18-29$ & 736 & $6.20(0.96)$ & $<0.001$ & 5.79 (2.35) & $<0.001$ & $10.3(1.47)$ & 0.001 & $6.46(0.86)$ & 0.402 \\
\hline $30-49$ & 1307 & $6.45(0.81)$ & & $6.26(2.30)$ & & $10.5(1.13)$ & & $6.51(0.80)$ & \\
\hline$\geq 50$ & 74 & $6.41(0.74)$ & & $7.01(2.48)$ & & $10.6(0.94)$ & & $6.46(0.98)$ & \\
\hline \multicolumn{10}{|l|}{ Ethnicity } \\
\hline Malay & 1381 & $6.33(0.85)$ & $<0.001$ & $5.85(2.30)$ & $<0.001$ & $10.37(1.32)$ & 0.045 & $6.49(0.80)$ & 0.586 \\
\hline Chinese & 430 & $6.59(0.74)$ & & $7.17(2.14)$ & & $10.46(1.14)$ & & $6.45(0.83)$ & \\
\hline Indian & 117 & $6.03(1.05)$ & & $6.11(2.52)$ & & $10.57(0.92)$ & & $6.50(0.92)$ & \\
\hline Others & 189 & $6.32(1.00)$ & & $5.74(2.30)$ & & $10.59(1.17)$ & & $6.54(0.97)$ & \\
\hline \multicolumn{10}{|l|}{ Education } \\
\hline $\begin{array}{l}\text { Primary or } \\
\text { below }\end{array}$ & 7 & $5.57(0.79)$ & $<0.001$ & $4.71(2.23)$ & $<0.001$ & $10.71(0.76)$ & 0.815 & $6.43(0.79)$ & 0.919 \\
\hline Secondary & 240 & $5.78(0.98)$ & & $4.60(2.15)$ & & $10.43(1.35)$ & & $6.47(0.88)$ & \\
\hline Tertiary & 1870 & $6.44(0.82)$ & & $6.32(2.29)$ & & $10.41(1.25)$ & & $6.49(0.82)$ & \\
\hline \multicolumn{10}{|l|}{ Occupation } \\
\hline $\begin{array}{c}\text { Civil } \\
\text { servant }\end{array}$ & 730 & $6.54(0.72)$ & $<0.001$ & $6.82(2.26)$ & $<0.001$ & $10.48(1.18)$ & $<0.001$ & $6.58(0.71)$ & 0.001 \\
\hline Private & 915 & $6.29(0.96)$ & & $5.87(2.32)$ & & $10.39(1.30)$ & & $6.43(0.90)$ & \\
\hline Student & 151 & $6.23(0.93)$ & & $5.68(2.24)$ & & $9.90(1.83)$ & & $6.36(0.84)$ & \\
\hline Unemployed & 303 & $6.25(0.82)$ & & $5.41(2.24)$ & & $10.61(0.83)$ & & $6.53(0.78)$ & \\
\hline Retiree & 18 & $6.17(0.86)$ & & $6.67(2.25)$ & & $10.50(0.79)$ & & $6.22(1.63)$ & \\
\hline \multicolumn{10}{|l|}{$\begin{array}{l}\text { Medical } \\
\text { education }\end{array}$} \\
\hline Yes & 590 & $6.58(0.70)$ & $<0.001$ & 7.69 (1.94) & $<0.001$ & $10.57(1.01)$ & $<0.001$ & $6.54(0.75)$ & 0.059 \\
\hline No & 1527 & $6.28(0.91)$ & & $5.52(2.20)$ & & $10.36(1.34)$ & & $6.47(0.86)$ & \\
\hline
\end{tabular}


Table A1. Cont.

\begin{tabular}{|c|c|c|c|c|c|c|c|c|c|}
\hline \multirow[t]{2}{*}{ Variable } & \multirow[t]{2}{*}{ Freq (n) } & \multicolumn{2}{|c|}{$\begin{array}{l}\text { Knowledge on } \\
\text { COVID-19 }\end{array}$} & \multicolumn{2}{|c|}{$\begin{array}{c}\text { Knowledge on Antibiotics } \\
\text { Resistance }\end{array}$} & \multicolumn{2}{|c|}{$\begin{array}{c}\text { Practice of Preventive } \\
\text { Measures }\end{array}$} & \multicolumn{2}{|c|}{$\begin{array}{c}\text { Attitude towards New } \\
\text { Norm }\end{array}$} \\
\hline & & Mean (SD) & $p$-Value & Mean (SD) & $p$-Value & Mean (SD) & $p$-Value & Mean (SD) & $p$-Value \\
\hline \multicolumn{10}{|l|}{$\begin{array}{l}\text { Chronic } \\
\text { disease }\end{array}$} \\
\hline Yes & 273 & $6.48(0.74)$ & 0.008 & $6.39(2.22)$ & 0.041 & $10.43(1.21)$ & 0.826 & $6.56(0.81)$ & 0.147 \\
\hline No & 1844 & $6.35(0.89)$ & & $6.08(2.36)$ & & $10.41(1.26)$ & & $6.48(0.83)$ & \\
\hline \multicolumn{10}{|l|}{$\begin{array}{l}\text { Household } \\
\text { income }\end{array}$} \\
\hline$<\mathrm{RM} 4850$ & 1038 & $6.17(0.94)$ & $<0.001$ & $5.54(2.28)$ & $<0.001$ & $10.45(1.22)$ & 0.232 & $6.47(0.83)$ & 0.652 \\
\hline $\begin{array}{c}\text { RM } \\
4850-\mathrm{RM} \\
10,970\end{array}$ & 793 & $6.52(0.73)$ & & $6.56(2.28)$ & & $10.40(1.22)$ & & $6.51(0.81)$ & \\
\hline $\begin{array}{r}\geq \mathrm{RM} \\
10,971\end{array}$ & 286 & $6.65(0.77)$ & & 7.03 (2.18) & & $10.31(1.45)$ & & $6.50(0.87)$ & \\
\hline \multicolumn{10}{|l|}{ Region } \\
\hline Central & 844 & $6.40(0.80)$ & 0.004 & $5.99(2.25)$ & 0.001 & 10.39 (1.19) & 0.009 & $6.53(0.78)$ & 0.141 \\
\hline Northern & 563 & $6.41(0.85)$ & & $6.44(2.40)$ & & $10.49(1.15)$ & & $6.48(0.76)$ & \\
\hline Sothern & 250 & $6.18(1.01)$ & & $5.86(2.34)$ & & 10.25 (1.62) & & $6.39(1.01)$ & \\
\hline Eastern & 184 & $6.32(0.84)$ & & $6.30(2.33)$ & & $10.28(1.47)$ & & $6.43(0.99)$ & \\
\hline Borneo & 269 & $6.36(0.97)$ & & $5.99(2.50)$ & & 10.58 (1.09) & & $6.53(0.80)$ & \\
\hline Overall & & $6.36(0.87)$ & Range: 0-7 & $6.12(2.34)$ & Range: 0-10 & $10.42(1.26)$ & Range: 0-11 & $6.49(0.83)$ & Range: $0-7$ \\
\hline
\end{tabular}

\section{References}

1. World Health Organization. Weekly Epidemiological Update. Available online: https://www.who.int/publications/m/item/ weekly-epidemiological-update--17-november-2020 (accessed on 17 November 2020).

2. Centers for Disease Control and Prevention. How to Protect Yourself. Available online: https://www.cdc.gov/coronavirus/2019 -ncov/prepare/prevention.html (accessed on 17 November 2020).

3. Petersen, E.; Koopmans, M.; Go, U.; Hamer, D.H.; Petrosillo, N.; Castelli, F.; Storgaard, M.; Al Khalili, S.; Simonsen, L. Comparing SARS-CoV-2 with SARS-CoV and influenza pandemics. Lancet Infect. Dis. 2020, 20, e238-e244. [CrossRef]

4. Elengoe, A. Covid-19 outbreak in Malaysia. Osong Public Health Res. Perspect. 2020, 11, 93-100. [CrossRef] [PubMed]

5. Sukumaran, T. Coronavirus: Malaysia in Partial Lockdown from March 18 to Limit Outbreak South China Morning Post. 16 March 2020. Available online: https://www.scmp.com/week-asia/health-environment/article/3075456/coronavirus-malaysiasprime-minister-muhyiddin-yassin (accessed on 17 November 2020).

6. Overseas Security Advisory Council. Health Alert: Malaysia, Movement Restrictions in Selangor, Kuala Lumpur, and Putrajaya. Available online: https:/ / www.osac.gov/Country/Malaysia/Content/Detail/Report/26c75edc-31a3-45c7-b0b5-19e85bd89513 (accessed on 17 November 2020).

7. World Health Organization. The New Normal. Available online: https://www.who.int/malaysia/emergencies/COVID-19-inmalaysia/information/the-new-normal (accessed on 17 November 2020).

8. Ministry of Health COVID-19 Guidelines. Annex 26. COVID-19: Social Distancing Guidelines for Workplace, Homes and Individuals. Available online: http:/ /COVID-19.moh.gov.my/garis-panduan/garis-panduan-kkm (accessed on 17 November 2020).

9. Chu, D.K.; Akl, E.A.; Duda, S.; Solo, K.; Yaacoub, S.; Schünemann, H.J. Physical distancing, face masks, and eye protection to prevent person-to-person transmission of SARS-CoV-2 and COVID-19: A systematic review and meta-analysis. Lancet 2020, 395, 1973-1987. [CrossRef] [PubMed]

10. Wake, A.D. Knowledge, Attitude, Practice, and Associated Factors Regarding the Novel Coronavirus Disease 2019 (COVID-19) Pandemic. Infect. Drug Resist. 2020, 13, 3817-3832. [CrossRef] [PubMed]

11. Zhong, B.-L.; Luo, W.; Li, H.-M.; Zhang, Q.-Q.; Liu, X.-G.; Li, W.-T.; Li, Y. Knowledge, attitudes, and practices towards COVID-19 among Chinese residents during the rapid rise period of the COVID-19 outbreak: A quick online cross-sectional survey. Int. J. Biol. Sci. 2020, 16, 1745-1752. [CrossRef] [PubMed]

12. Al-Hanawi, M.K.; Angawi, K.; Alshareef, N.; Qattan, A.M.N.; Helmy, H.Z.; Abudawood, Y.; Alqurashi, M.; Kattan, W.M.; Kadasah, N.A.; Chirwa, G.C.; et al. Knowledge, attitude and practice toward Covid-19 among the public in the Kingdom of Saudi Arabia: A cross-sectional study. Front. Public Health 2020, 8, 217. [CrossRef] [PubMed] 
13. Huynh, G.; Nguyen, M.Q.; Tran, T.T.; Nguyen, V.T.; Nguyen, T.V.; Do, T.H.T.; Nguyen, P.H.N.; Phan, T.H.Y.; Vu, T.T.; Nguyen, T.N.H. Knowledge, attitude, and practices regarding COVID-19 among chronic illness patients at outpatient departments in Ho Chi Minh City, Vietnam. Risk Manag. Healthc. Policy 2020, 13, 1571-1578. [CrossRef] [PubMed]

14. Qutob, N.; Awartani, F. Knowledge, attitudes and practices (KAP) towards COVID-19 among Palestinians during the COVID-19 outbreak: A cross-sectional survey. PLoS ONE 2021, 16, e0244925. [CrossRef]

15. Al Ahdab, S. A cross-sectional survey of knowledge, attitude and practice (KAP) towards COVID-19 pandemic among the Syrian residents. BMC Public Health 2021, 21, 296. [CrossRef]

16. Afzal, M.S.; Khan, A.; Qureshi, U.U.R.; Saleem, S.; Saqib, M.A.N.; Shabbir, R.M.K.; Naveed, M.; Jabbar, M.; Zahoor, S.; Ahmed, H. Community-Based Assessment of Knowledge, Attitude, Practices and Risk Factors Regarding COVID-19 Among Pakistanis Residents During a Recent Outbreak: A Cross-Sectional Survey. J. Community Health 2020, 1-11. [CrossRef]

17. Haque, T.; Hossain, K.M.; Bhuiyan, M.d.M.R.; Ananna, S.A.; Chowdhury, S.H.; Islam, M.R.; Ahmed, A.; Rahman, M.M. Knowledge, Attitude and Practices (KAP) towards COVID-19 and Assessment of Risks of Infection by SARS-CoV-2 among the Bangladeshi Population: An Online Cross Sectional Survey [Internet]. In Review; April 2020. Available online: https: //www.researchsquare.com/article/rs-24562/v1 (accessed on 17 November 2020).

18. Lau, L.L.; Hung, N.; Go, D.J.; Ferma, J.; Choi, M.; Dodd, W.; Wei, X. Knowledge, attitudes and practices of COVID-19 among income-poor households in the Philippines: A cross-sectional study. J. Glob. Health 2020, 10, 011007. [CrossRef] [PubMed]

19. Azlan, A.A.; Hamzah, M.R.; Sern, T.J.; Ayub, S.H.; Mohamad, E. Public knowledge, attitudes and practices towards COVID-19: A cross-sectional study in Malaysia. PLoS ONE 2020, 15, e0233668. [CrossRef] [PubMed]

20. Thomas, R.; Greenwood, H.; Michaleff, Z.A.; Abukmail, E.; Hoffmann, T.C.; McCaffery, K.; Hardiman, L.; Glasziou, P. Examining Australian's beliefs, misconceptions and sources of information for COVID-19: A national online survey. BMJ Open 2021, 11, e043421. [CrossRef] [PubMed]

21. Langford, B.J.; So, M.; Raybardhan, S.; Leung, V.; Westwood, D.; MacFadden, D.R.; Soucy, J.-P.R.; Daneman, N. Bacterial co-infection and secondary infection in patients with COVID-19: A living rapid review and meta-analysis. Clin. Microbiol. Infect. 2020, 26, 1622-1629. [CrossRef] [PubMed]

22. Ab Halim, N.A.A.; Chang, C.-T.; Chan, H.K.; Hassali, M.A.; Nouri, A. Knowledge and Attitudes Concerning Antibiotic Use and Resistance among the Public in Pulau Pinang, Malaysia. Malays. J. Med. Sci. 2018, 25, 141-147. [CrossRef] [PubMed]

23. Choo, S.J.; Chang, C.T.; Lee, J.C.Y.; Munisamy, V.; Tan, C.K.; Raj, J.D.; Taib, R.I.M.; Thong, K.S.; Shafie, A.A. A cross-sectional study on public belief, knowledge and practice towards antibiotic use in the state of Perak, Malaysia. J. Infect. Dev. Ctries. 2018, 12, 960-969. [CrossRef] [PubMed]

24. Mallah, N.; Badro, D.A.; Figueiras, A.; Takkouche, B. Association of knowledge and beliefs with the misuse of antibiotics in parents: A study in Beirut (Lebanon). PLoS ONE 2020, 15, e0232464. [CrossRef] [PubMed]

25. Byambasuren, O.; Cardona, M.; Bell, K.; Clark, J.; McLaws, M.-L.; Glasziou, P. Estimating the extent of asymptomatic COVID-19 and its potential for community transmission: Systematic review and meta-analysis. Off. J. Assoc. Med. Microbiol. Infect. Dis. Can. 2020, 5, 223-234. [CrossRef]

26. He, D.; Zhao, S.; Lin, Q.; Zhuang, Z.; Cao, P.; Wang, M.H.; Yang, L. The relative transmissibility of asymptomatic COVID-19 infections among close contacts. Int. J. Infect. Dis. 2020, 94, 145-147. [CrossRef] [PubMed]

27. Guan, W.J.; Liang, W.H.; Zhao, Y.; Liang, H.-R.; Chen, Z.-S.; Li, Y.-M.; Liu, X.-Q.; Chen, R.-C.; Tang, C.-L.; Wang, T.; et al. Comorbidity and its impact on 1590 patients with COVID-19 in China: A nationwide analysis. Eur. Respir. J. 2020, 55, 2000547. [CrossRef]

28. Rawson, T.M.; Moore, L.S.P.; Zhu, N.; Ranganathan, N.; Skolimowska, K.; Gilchrist, M.; Satta, G.; Cooke, G.; Holmes, A. Bacterial and Fungal Coinfection in Individuals with Coronavirus: A Rapid Review to Support COVID-19 Antimicrobial Prescribing. Clin. Infect. Dis. 2020, 71, 2459-2468. [CrossRef] [PubMed]

29. Strathdee, S.A.; Davies, S.C.; Marcelin, J.R. Confronting antimicrobial resistance beyond the COVID-19 pandemic and the 2020 US election. Lancet 2020, 396, 1050-1053. [CrossRef] [PubMed]

30. Mouhieddine, T.H.; Olleik, Z.; Itani, M.M.; Kawtharani, S.; Nassar, H.; Hassoun, R.; Houmani, Z.; El Zein, Z.; Fakih, R.; Mortada, I.K.; et al. Assessing the Lebanese population for their knowledge, attitudes and practices of antibiotic usage. J. Infect. Public Health 2015, 8, 20-31. [CrossRef] [PubMed]

31. Nepal, A.; Hendrie, D.; Robinson, S.; Selvey, L.A. Knowledge, attitudes and practices relating to antibiotic use among community members of the Rupandehi District in Nepal. BMC Public Health 2019, 19, 1558. [CrossRef] [PubMed]

32. Jairoun, A.; Hassan, N.; Ali, A.; Jairoun, O.; Shahwan, M. Knowledge, attitude and practice of antibiotic use among university students: A cross sectional study in UAE. BMC Public Health 2019, 19, 518. [CrossRef] [PubMed]

33. Akalu, Y.; Ayelign, B.; Molla, M.D. Knowledge, attitude and practice towards COVID-19 among chronic disease patients at Addis Zemen Hospital, Northwest Ethiopia. Infect. Drug Resist. 2020, 13, 1949-1960. [CrossRef] [PubMed]

34. World Health Organization. Shortage of Personal Protective Equipment Endangering Health Workers Worldwide. Available online: https:/ / www.who.int/news/item/03-03-2020-shortage-of-personal-protective-equipment-endangering-health-workersworldwide (accessed on 17 November 2020).

35. Ministry of Health Malaysia. Annex 8a: Guidance on the Use of Masks with Regards to COVID-19 Pandemic. Available online: http:/ /COVID-19.moh.gov.my/garis-panduan/garis-panduan-kkm/Annex_8a_RECOMENDATIONS_ON_FACE_ MASK.pdf (accessed on 17 November 2020). 
36. World Health Organization. When and How to Use Masks. Available online: https:/ /www.who.int/emergencies / diseases / novel-coronavirus-2019/advice-for-public/when-and-how-to-use-masks (accessed on 17 November 2020).

37. Kang, S.-J.; Jung, S.I. Age-related morbidity and mortality among patients with Covid-19. Infect. Chemother. 2020, 52, 154. [CrossRef]

38. Jin, J.; Sklar, G.E.; Oh, V.M.S.; Li, S.C. Factors affecting therapeutic compliance: A review from the patient's perspective. Ther. Clin. Risk Manag. 2008, 4, 269-286.

39. Marmot, M. The Influence of Income on Health: Views of an Epidemiologist. Health Aff. 2002, 21, 31-46. [CrossRef] [PubMed]

40. From the Desk of the Director General of Health Malaysia. Available online: https://kpkesihatan.com/ (accessed on 17 November 2020).

41. The National Security Council. Available online: https://www.mkn.gov.my/web/ms/ (accessed on 17 November 2020).

42. KPMG. The Work from Home Resolution. Available online: https://home.kpmg/my/en/home/insights/2020/03/the-businessimplications-of-coronavirus/the-work-from-home-revolution.html (accessed on 17 November 2020).

43. Bonacini, L.; Gallo, G.; Scicchitano, S. Working from home and income inequality: Risks of a 'new normal' with COVID-19. J. Popul. Econ. 2021, 34, 303-360. [CrossRef] 